\title{
New chaotic planar attractors from smooth zero entropy interval maps
}

\author{
Jan P Boroński ${ }^{1,2^{*}}$ and Jiří Kupka'
}

\author{
"Correspondence: \\ jan.boronski@osu.cz \\ ${ }^{1}$ National Supercomputing Centre \\ IT4Innovations, Division of the \\ University of Ostrava, Institute for \\ Research and Applications of Fuzzy \\ Modeling, 30. Dubna 22, Ostrava, \\ 701 03, Czech Republic \\ ${ }^{2}$ Faculty of Applied Mathematics, \\ AGH University of Science and \\ Technology, al. Mickiewicza 30, \\ Kraków, 30-059, Poland
}

\begin{abstract}
We show that for every positive integer $k$ there exists an interval map $f: I \rightarrow I$ such that (1) $f$ is Li-Yorke chaotic, (2) the inverse limit space $I_{f}=\lim _{\leftarrow}\{f, /\}$ does not contain an indecomposable subcontinuum, (3) $f$ is $C^{k}$-smooth, and (4) $f$ is not $C^{k+1}$-smooth. We also show that there exists a $C^{\infty}$-smooth $f$ that satisfies (1) and (2). This answers a recent question of Oprocha and the first author from (Proc. Am. Math. Soc. 143(8):3659-3670, 2015), where the result was proved for $k=0$. Our study builds on the work of Misiurewicz and Smítal of a family of zero entropy weakly unimodal maps. With the help of a result of Bennett, as well as Blokh's spectral decomposition theorem, we are also able to show that each $I_{f}$ contains, for every integer $i$, a subcontinuum $C_{i}$ with the following two properties: (i) $C_{i}$ is $2^{i}$-periodic under the shift homeomorphism, and (ii) $C_{i}$ is a compactification of a topological ray. Finally, we prove that the chaotic attractors we construct are topologically distinct from the one presented by P Oprocha and the first author.
\end{abstract}

MSC: 54H20; 37B45; 37E05

Keywords: weakly unimodal map; arc-like; Li-Yorke chaotic; indecomposable continuum; zero topological entropy

\section{Introduction}

The celebrated Cartwright-Littlewood-Bell fixed point theorem [1, 2] asserts that any homeomorphism of $\mathbb{R}^{2}$ must fix a point in an invariant plane nonseparating compact and connected set (continuum). The original work of Cartwright and Littlewood, motivated by problems in differential equations, in which they were led to consider invariant sets whose frontiers were indecomposable, brought more focus in the mathematical literature to the interplay of topology and dynamics in continua, of which the study of planar attractors generated by inverse limits of arcs became an important theme. The well known connection established by Barge and Martin in the 1980s states that chaotic (in the sense of positive entropy) interval maps generate planar dynamical systems with attractors that must contain an indecomposable subcontinuum (see e.g. [3, 4]). The connection is in fact a characterization for all piecewise monotone graph maps [5], but there had also been an aspect of it left over: must weak chaos (i.e. chaos in the sense of Li and Yorke) imply indecomposability in the inverse limit space? Recently, however, Oprocha and the first author showed in [6] that there exists a Li-Yorke chaotic interval map $F$ such that the inverse limit space $I_{F}=\lim _{\leftarrow}\{F, I\}$ does not contain an indecomposable subcontinuum. Since the

C 2015 Boroński and Kupka. This article is distributed under the terms of the Creative Commons Attribution 4.0 International License (http://creativecommons.org/licenses/by/4.0/), which permits unrestricted use, distribution, and reproduction in any medium, provided you give appropriate credit to the original author(s) and the source, provide a link to the Creative Commons license, and indicate if changes were made. 
map $F$ constructed in [6] was not continuously differentiable, the following question was posed.

Problem 1 [6] Is there a $k>0$ and a Li-Yorke chaotic interval map $\varphi$ such that $\varphi$ is $C^{k}$-smooth and the inverse limit space $I_{\varphi}=\lim _{\leftarrow}\{\varphi, I\}$ does not contain an indecomposable subcontinuum? Must $I_{\varphi}$ have a periodic structure similar to the continua described in $[6]$ ?

In the present paper, by employing an entirely different approach, we answer the question in the affirmative. First we prove the following result.

Theorem 1 For every positive integer $k$ there exists an interval map $f: I \rightarrow I$ such that

(1) $f$ is Li-Yorke chaotic,

(2) $I_{f}=\lim _{\leftarrow}\{f, I\}$ does not contain an indecomposable subcontinuum,

(3) $f$ is $C^{k}$-smooth,

(4) $f$ is not $C^{k+1}$-smooth.

Theorem 2 There exists a $C^{\infty}$-smooth interval map $f: I \rightarrow I$ such that

(1) $f$ is Li-Yorke chaotic,

(2) $I_{f}=\lim _{\leftarrow}\{f, I\}$ does not contain an indecomposable subcontinuum.

It is noteworthy to mention that the Li-Yorke chaotic map $F$ without an indecomposable subcontinuum in $\lim _{\leftarrow}\{F, I\}$ constructed in [6] was not piecewise monotone, having countably many intervals of monotonicity. It was shown therein that, by an arbitrarily small perturbation, the map could be modified without increasing entropy to a map $F_{\epsilon}$, as to generate various indecomposable subcontinua in $\lim _{\leftarrow}\left\{F_{\epsilon}, I\right\}$, including Knaster's pseudo-arc. An appropriate perturbation $F_{S}$ could also result in the attractor $\lim _{\leftarrow}\left\{F_{S}, I\right\}$ being non-Suslinean. Our approach is different, as it is based on a careful selection of weakly unimodal maps. Although, in general, such maps may have positive entropy, and therefore may generate indecomposable subcontinua of their inverse limit spaces, there is a class of zero entropy and Li-Yorke chaotic weakly unimodal maps. The class was studied by Misiurewicz and Smítal [7] who, among other results, showed that the class contains a nonempty subclass of $C^{\infty}$-smooth maps (see Section 2). In Section 3, we shall observe that the class also contains maps with other degrees of differentiability. In addition, since weakly unimodal maps are piecewise monotone, we shall be able to use the result of Barge and Diamond [5] that relates entropy of piecewise monotone graph maps to topological structure of the related inverse limit spaces (see next section for more details). In Section 4 we further study the topological structure of our attractors. We show that they have a similar periodic structure to the attractors in [6].

Theorem 3 Suppose $f: I \rightarrow I$ is a Li-Yorke chaotic zero entropy weakly unimodal map. Then $I_{f}$ contains, for every $i$, a subcontinuum $C_{i}$ with the following two properties:

(i) $C_{i}$ is $2^{i}$-periodic under the shift homeomorphism, and

(ii) $C_{i}$ is a compactification of a topological ray.

The proof of the above theorem, as in [6], will rely on a careful application of Bennett's theorem (see [8]) but also Blokh's spectral decomposition theorem [9]. However, we also show that the chaotic attractors we construct are topologically distinct from those in [6] 


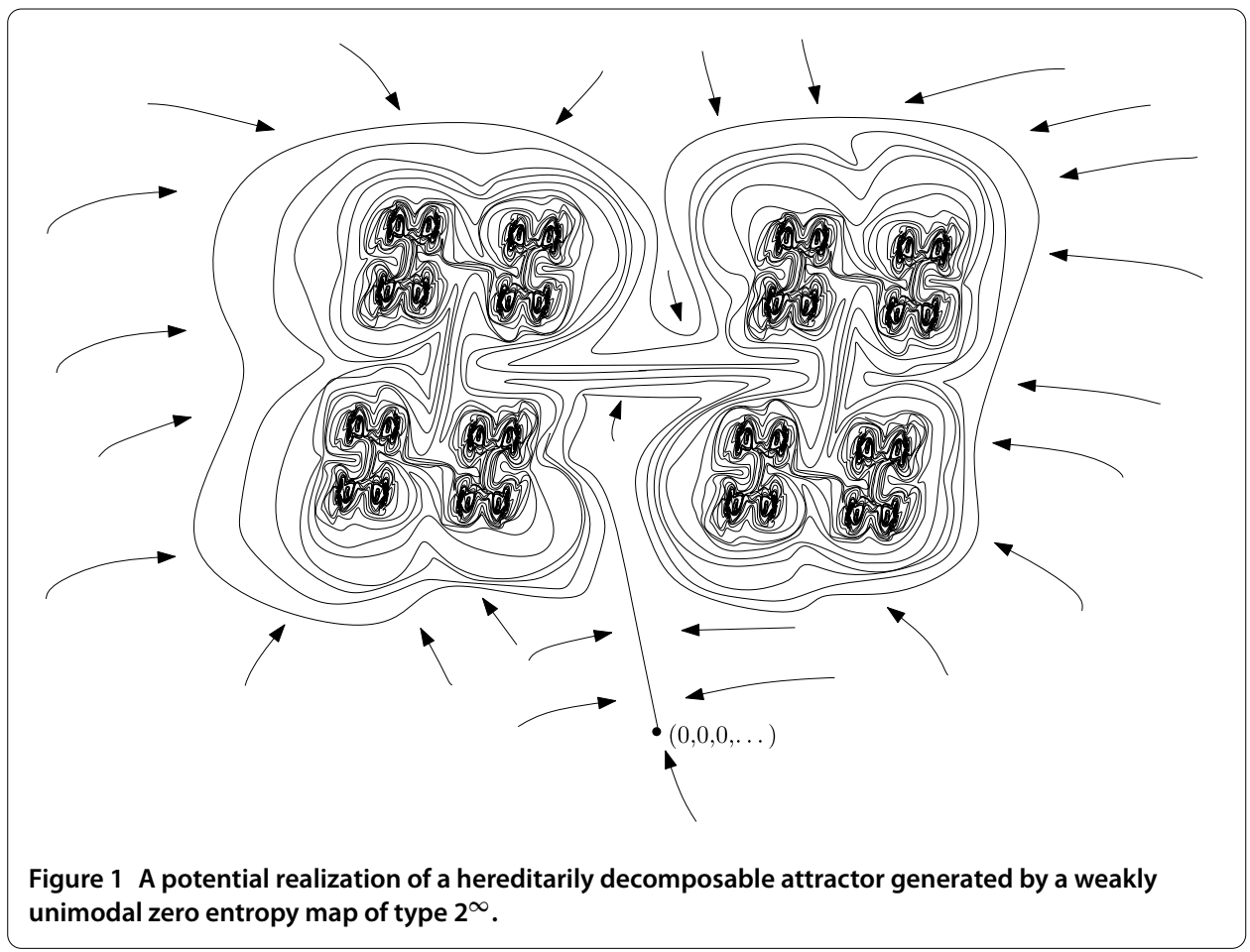

(a potential realization of one of our attractors is depicted in Figure 1). We conclude our paper stating some questions related to the study of Li-Yorke chaotic zero entropy interval maps.

\section{Preliminaries}

By $I$ we shall denote the unit interval $[0,1]$. A map is a continuous function. A continuum $X$ is a compact and connected metric space that contains at least two points. A subcontinuum of a continuum $X$ is a continuum contained in $X$ as a subset. A class of maps $f: X \rightarrow X$ is denoted by $C(X)$. A continuum is decomposable if it cannot be written as the union of two proper subcontinua. It is hereditarily decomposable if every subcontinuum is decomposable. An indecomposable continuum is a continuum that is not decomposable, and it is hereditarily indecomposable if every subcontinuum is indecomposable. A continuum is said to be Suslinean provided every family of pairwise disjoint subcontinua is at most countable. Although all indecomposable continua are not Suslinean, a decomposable continuum may or may not be Suslinean. A space $X$ is arcwise connected if for every two points $x$ and $y$ in $X$ there is an $\operatorname{arc} A \subseteq X$ such that $x, y \in A$. A (topological) ray is a homeomorphic image of the half-line $[0,+\infty)$ and a (topological) line is a homeomorphic image of $(-\infty,+\infty)$. Suppose a map $f: I \rightarrow I$ is given. The inverse limit space $I_{f}=\lim _{\leftarrow}\{f, I\}$ is the space given by

$$
I_{f}=\left\{\left(x_{1}, x_{2}, x_{3}, \ldots\right) \in I^{\mathbb{N}}: f\left(x_{i+1}\right)=x_{i}\right\} .
$$

The topology of $I_{f}$ is induced from the product topology of $I^{\mathbb{N}}$, with the basic open sets in $I_{f}$ given by

$$
U_{\leftarrow}=\left(f^{i-1}(U), f^{i-2}(U), \ldots, U, f^{-1}(U), f^{-2}(U), \ldots\right),
$$


where $U$ is an open subset of the $i$ th factor space $I$, and $i$ ranges over $\mathbb{N}$ (see e.g. Theorem 3 on p.79 in [10]). It is well known that $I_{f}$ is a continuum, and it is called an arc-like (or chainable) continuum. The pseudo-arc is the unique hereditarily indecomposable arc-like continuum (see [11] for more details on the pseudo-arc). There is a natural homeomorphism $\sigma_{f}: I_{f} \rightarrow I_{f}$, called the shift homeomorphism, given by

$$
\sigma_{f}\left(x_{1}, x_{2}, x_{3}, \ldots\right)=\left(f\left(x_{1}\right), f\left(x_{2}\right), f\left(x_{3}\right), \ldots\right)=\left(f\left(x_{1}\right), x_{1}, x_{2}, \ldots\right) .
$$

The shift homeomorphism $\sigma_{f}$ preserves topological entropy of $f$, as well as many other dynamical properties such as existence of periodic orbits of given period, shadowing property, and topological mixing [12]. Barge and Martin proved [4] that any $I_{f}$ embeds in $\mathbb{R}^{2}$ in such a way that there exists a planar homeomorphism $h$ with the following properties:

- $h\left(I_{f}\right)=I_{f}$,

- $\left.h\right|_{I_{f}}=\sigma_{f}$ (up to conjugacy), and

- there is an open set $N$ containing $I_{f}$ such that $\bigcap_{n=1}^{\infty} h^{n}(N)=I_{f}$.

For this reason we shall refer to any $I_{f}$ as an arc-like attractor. Let $\rho$ denote the metric on $X$. A map $\varphi: X \rightarrow X$ is Li-Yorke chaotic if there is an uncountable set $S \subset X$ such that

$$
\liminf _{n \rightarrow \infty} \rho\left(\varphi^{n}(x), \varphi^{n}(y)\right)=0
$$

and

$$
\limsup _{n \rightarrow \infty} \rho\left(\varphi^{n}(x), \varphi^{n}(y)\right)>0
$$

for any distinct points $x, y \in S$. An interval map $f$ is called unimodal if there exists a turning point $c \in I, 0<c<1$, such that $\left.f\right|_{[0, c]}$ is strictly increasing and $\left.f\right|_{[c, 1]}$ is strictly decreasing. A map $f$ is weakly unimodal if there exists a $c \in I, 0<c<1$, such that $\left.f\right|_{[0, c]}$ is nondecreasing and $\left.f\right|_{[c, 1]}$ is nonincreasing. We say that an interval map (or graph map) $f: G \rightarrow f(G)$ is monotone if $f^{-1}(x)$ is connected for every $x \in f(G)$. We say that $f$ is piecewise monotone on $G$ if there is a finite set of points $A=\left\{a_{1}, \ldots, a_{n}\right\} \subseteq G$ such that $f$ is monotone on each component of $G \backslash A$. Note that every weakly unimodal map is piecewise monotone.

Let us introduce Bowen's definition of the topological entropy (see [13]). Let $K \subset X$ be a compact subset, and fix $\varepsilon>0$ and $n \in \mathbb{N}$. We say that a set $E \subset K$ is $(n, \varepsilon, K, f)$-separated (by the map $f)$ if for any $x, y \in E, x \neq y$, there is $k \in\{0,1, \ldots, n-1\}$ such that $\rho\left(f^{k}(x), f^{k}(y)\right)>\varepsilon$. Denote by $s_{n}(\varepsilon, K, f)$ the cardinality of any maximally $(n, \varepsilon, K, f)$-separated set in $K$ and define

$$
s(\varepsilon, K, f)=\limsup _{n \rightarrow \infty} \frac{1}{n} \log s_{n}(\varepsilon, K, f) .
$$

Then the topological entropy of $f$ is

$$
h(f)=\sup _{K} \lim _{\varepsilon \rightarrow 0} s(\varepsilon, K, f) .
$$

Finally, we say that two discrete dynamical systems $(X, f)$ and $(Y, g)$ are conjugate if there exists a continuous bijection $h: X \rightarrow Y$ (called a conjugacy) for which $h \circ f=g \circ h$. If $h$ 
is only a continuous surjection then we have a semiconjugacy. It is well known that the topological entropy is invariant with respect to a conjugacy.

The following result of Barge and Diamond will be important to our results. Note that interval maps are special cases of graph maps.

Lemma 4 (Barge and Diamond [5]) Suppose $f: G \rightarrow G$ is a piecewise monotone graph map. $f$ has zero topological entropy if and only if $\lim _{\leftarrow}\{f, G\}$ does not contain an indecomposable subcontinuum.

Let us consider a system $\mathcal{F} \subseteq C(I)$ of weakly unimodal interval maps satisfying the following conditions:

(1) the set

$$
J_{f}:=\{x \in I \mid f(y) \leq f(x) \text { for every } y \in I\}
$$

consists of more than one point,

(2) for each $n \in \mathbb{N}, f$ has a periodic point of period $2^{n}$,

(3) $f$ has no periodic points of other periods.

The family $\mathcal{F}$ is nonempty and any map that satisfies the properties (2) and (3) is said to be of type $2^{\infty}$. In [7] the following two results are proved (see also [14] for an alternative proof of the first result, and the comments on p.674 in [15] concerning [7]).

Lemma 5 [7] Any map $f \in \mathcal{F}$ has zero topological entropy and is chaotic in the sense of $L i$ and Yorke.

Lemma 6 [7] Let $\mathcal{F}_{0} \subseteq \mathcal{F}$ be a family of $C^{\infty}$ interval maps $f$ satisfying $f(0)=f(1)=0$. Then $\mathcal{F}_{0} \neq \emptyset$.

\section{Main results}

With the help of the following result we are able to ensure the existence of the desired Li-Yorke chaotic map in each differentiability class that we claim in our main theorems. Our proof of (iv) of the next result closely follows that of Theorem 3 in [7].

Lemma 7 For every positive integer $k$ there exists a weakly unimodal map $f:[0,1] \rightarrow[0,1]$ such that

(i) $f(0)=f(1)=0$,

(ii) $f$ is $C^{k}$-smooth,

(iii) $f$ is not $C^{k+1}$-smooth.

Moreover, for any $c \in(0,1]$, the map $c \cdot f$ satisfies (i)-(iii) and

(iv) there exists $\bar{c} \in(0,1]$ such that $\bar{c} \cdot f \in \mathcal{F}$.

Proof Let $e_{1}, e_{2} \in I$ be such that $0<e_{1}<e_{2}<1$ and let $f:\left[0, e_{1}\right] \rightarrow[0,1]$ be given by

$$
f(x)=\left[\exp \left(e_{1}^{k}\right)-\exp \left(\left|x-e_{1}\right|^{k}\right)\right] /\left[\exp \left(e_{1}^{k}\right)-1\right]
$$

It is not difficult to see that $f$ is strictly monotone, $f(0)=0, f\left(e_{1}\right)=1$, and $f^{(i)}\left(e_{1}\right)=0$ if and only if $i=1, \ldots, k-1$. Setting $f(x)=1$ for every $x \in\left[e_{1}, e_{2}\right]$ and defining $f$ on $\left[e_{2}, 1\right]$ 
by symmetry, analogously to $\left[0, e_{1}\right]$. Note that $f$ is not $C^{k+1}$-smooth as $f^{(k+1)}\left(e_{1}\right)$ (and also $\left.f^{(k+1)}\left(e_{2}\right)\right)$ does not exist. Therefore, we get the properties (i)-(iii).

Let us prove (iv). It is easy to see that, for any $c \in(0,1], \varphi_{c}:=c \cdot f$ is a weakly unimodal map and that $J_{\varphi_{c}}$ is a nondegenerate interval (by the choice of $e_{1}$ and $e_{2}$ ). It remains to show that $\varphi_{\bar{c}}$ is of type $2^{\infty}$ for some $\bar{c}$.

It is well known that $h\left(\varphi_{1}\right)>0$ and the smaller $c$ is, the smaller $h\left(\varphi_{c}\right)$ we can get. For a sufficiently small $c$, periodic points of $\varphi_{c}$ are just fixed points and, hence, $h\left(\varphi_{c}\right)=0$. Consequently, $M:=\left\{c \in(0,1] \mid h\left(\varphi_{c}\right)>0\right\}$ is nonempty and $\bar{c}=\inf M>0$.

It is well known (see e.g. [16]) that $\varphi_{c} \in M$ implies that $\varphi_{c} \in N$ where $N:=\{f \in I \rightarrow I \mid$ $f$ satisfies (2) of the definition of $\mathcal{F}$ \}. By Proposition 2.1 in [17], the set $N$ is closed, since its elements are $C^{1}$. Therefore, $\varphi_{\bar{c}} \in N$. We also claim that $\varphi_{\bar{c}}$ satisfies (3) of the definition of $\mathcal{F}$. Otherwise, $\varphi_{\bar{c}}$ would have a periodic point of period other than $2^{n}$, and hence (again [16]) $h\left(\varphi_{\bar{c}}\right)>0$. But this would contradict the choice of $\bar{c}$, because the set $M$ is open (see e.g. [18]). This completes the proof.

Note that the above approach depends on the differentiability of the maps in question and it does not work for tent maps; i.e. maps of the form

$$
f_{c}(x)=c \cdot \min \{x, 1-x\}
$$

as they are not $C^{1}$. Indeed, there is no tent map of type $2^{\infty}$, because such a map would have to have zero entropy, and this is only possible for $c \in[0,1]$ (see e.g. [19]), but for no such $c$ the $\operatorname{map} f_{c}$ has even a 2-periodic point. ${ }^{\mathrm{a}}$ On the other hand, by Lemma 5 there is an $M$ such that the truncated full tent $\operatorname{map} g(x)=\min \left\{M, f_{2}(x)\right\}$ is a weakly unimodal Li-Yorke chaotic $C^{0}$, but not $C^{1}$, map of type $2^{\infty}$. Now we are ready to prove our main results.

Theorem 1 For every positive integer $k$ there exists an interval map $f: I \rightarrow I$ such that

(1) $f$ is Li-Yorke chaotic,

(2) $I_{f}=\lim _{\leftarrow}\{f, I\}$ does not contain an indecomposable subcontinuum,

(3) $f$ is $C^{k}$-smooth,

(4) $f$ is not $C^{k+1}$-smooth.

Proof of Theorem 1 Let $k \in \mathbb{N}$ be fixed and let $f$ be a map guaranteed by Lemma 7. Since $f \in \mathcal{F}$ we see that $f$ is also Li-Yorke chaotic and has zero entropy. Since $f$ is piecewise monotone and has zero entropy, by Lemma 4 , we also see that $\lim _{\leftarrow}\{f, I\}$ does not contain an indecomposable subcontinuum.

Theorem 2 There exists a $C^{\infty}$-smooth interval map $f: I \rightarrow I$ such that

(1) $f$ is Li-Yorke chaotic,

(2) $I_{f}=\lim _{\leftarrow}\{f, I\}$ does not contain an indecomposable subcontinuum.

Proof of Theorem 2 By Lemmas 5 and 6 there is a $C^{\infty}$-smooth, Li-Yorke chaotic map $f$ with zero entropy. Again, by Lemma 4, the inverse limit space $\lim _{\leftarrow}\{f, I\}$ does not contain an indecomposable subcontinuum.

At the end of this section we note that all the attractors obtained by Theorem 1 and Theorem 2 are Suslinean by main Theorem 1 in [20]. As a consequence each of them 
can be embedded into the plane in such a way that all its points are accessible from the complement, by the results in [21].

\section{More on the topological structure of the attractors}

Although the attractors constructed in the present paper have a similar periodic structure to the two attractors constructed in [6] (see Theorem 12), they are topologically distinct from the latter. We shall show that the attractors described in the present paper in Theorem 1 and Theorem 2 are compactifications of a ray, whereas the attractors in [6] are not. As in [6], we shall use the following result of Bennett.

Theorem 8 (Bennett) (the proof can be found in [8]) Suppose that $g:[a, b] \rightarrow[a, b]$ is continuous and $d \in(a, b)$ is such that

(1) $g([d, b]) \subset[d, b]$,

(2) $\left.g\right|_{[a, d]}$ is monotone, and

(3) there is $n \in \mathbb{N}$ such that $g^{n}([a, d])=[a, b]$.

Then continuum $K=\lim _{\leftarrow}\{g,[a, b]\}$ is the union of a topological ray $R$ and a continuum $C=\lim _{\leftarrow}\{g,[d, b]\}$ such that $\bar{R} \backslash R=C$.

Lemma 9 For every $f \in \mathcal{F}$ with $f(0)=0$ there are $a, b \in I$ and $d \in(a, b)$ such that

(1) $f([d, b]) \subset[d, b]$,

(2) $\left.f\right|_{[a, d]}$ is monotone, and

(3) there is an $n \in \mathbb{N}$ such that $f^{n}([a, d])=[a, b]$.

Proof Let $f \in \mathcal{F}$ be fixed. By (1) of the definition of $\mathcal{F}$, there exists a nondegenerate interval $\left[e_{1}, e_{2}\right]$ such that $f(x)=m=\max _{y \in I}\{f(y)\}$ for each $x \in\left[e_{1}, e_{2}\right]$.

Because $f\left(e_{2}\right)=m$ is the maximum of $f$ and $\left[e_{1}, e_{2}\right]$ contains no periodic point (see [7]), we have $e_{2}<f\left(e_{2}\right)<1$. Thus,

$$
f\left(f\left(e_{2}\right)\right)>f(1)
$$

since $f$ is nonincreasing on $\left[e_{2}, 1\right]$ and $f(1)<m$. Otherwise (if $f(1)=m$ ) all periodic points would be fixed points and this would contradict $f \in \mathcal{F}$.

Moreover,

$$
f\left(f\left(e_{2}\right)\right)>a:=\max \left\{x \in\left[0, e_{1}\right) \mid f(x)=x\right\} .
$$

Otherwise, for $U=\left[a, e_{1}\right], V=\left[e_{1}, f\left(e_{2}\right)\right]$, we would prove that $f$ has positive topological entropy (see [16]), because

$$
f(U) \cap f(V) \supseteq U \cup V .
$$

Because $\left.f\right|_{\left[0, e_{1}\right]}$ is nondecreasing, the choice of $a$ implies that there exists a strictly decreasing sequence $\left\{a_{i}\right\}_{i=1}^{\infty} \subseteq\left[a, e_{1}\right]$ converging to $a$ such that $a_{1}=e_{1}$ and $a_{i}=f\left(a_{i+1}\right)$ for $i \in \mathbb{N}$.

Now let $b:=m$ and $d:=\max \left\{\left\{a_{i}\right\}_{i \in \mathbb{N}} \cap[a, f(b))\right\}$. It follows from (1) that such a $d$ exists. Moreover,

$$
f(d) \in\left(f(b), e_{1}\right]
$$


Then, by the monotonicity of $f$ on $\left[d, e_{1}\right]$ and $\left[e_{1}, b\right]$,

$$
f([d, b])=f\left(\left[d, e_{1}\right] \cup\left[e_{1}, b\right]\right) \subseteq[f(b), m] \subseteq[d, b],
$$

i.e. the first property is checked. The second property follows from the fact that $f$ is nondecreasing on $\left[0, e_{1}\right]$ and $[a, d] \subseteq\left[0, e_{1}\right]$. The last property follows from the fact that

$$
f\left(\left[a, a_{i+1}\right]\right)=\left[a, a_{i}\right]
$$

for any $i \in \mathbb{N}$, and from the facts that elements of $\left\{a_{i}\right\}_{i \in \mathbb{N}}$ are preimages of $e_{i}$ and $e_{i}$ is one of preimages of $b$.

By using the notation of the proof above, one can see that either $a=0$ or $a>0$ and we have $f([0, a])=[0, a]$. Thus, one can change $a$ to be equal to 0 and because $f^{-1}((m, 0])=\emptyset$ the following corollary of Theorem 8 can be obtained. So we strengthen Lemma 9.

Corollary 10 For every $f \in \mathcal{F}$ with $f(0)=0$, there is a topological ray $L$ such that $\bar{L}=I_{f}$.

Note that the proof of Lemma 9 holds the following result originally proved in [6], Remark 10 .

Corollary 11 There is a Li-Yorke chaotic zero entropy map $\varphi: I \rightarrow I$ such that $I_{\varphi}$ contains the pseudo-arc.

Proof Fix an $f \in \mathcal{F}$ and let $a, d$, and $m$ be as in the proof of Lemma 9. Let $f_{H}:[0, a] \rightarrow$ $[0, a]$ be the Henderson map [22], rescaled to $[0, a]$. Now set $\varphi(x)=f_{H}(x)$ for $x \in[0, a]$ and $\varphi(x)=f(x)$ for $x \in[a, 1]$.

Lemma 12 Suppose $f: I \rightarrow I$ is a map of type $2^{\infty}$. Then the shift homeomorphism $\sigma_{f}$ has a $2^{i}$-periodic subcontinuum of $I_{f}$, for every integer $i>0$.

Proof Fix an integer $i>0$. It follows from Blokh's spectral decomposition theorem (see e.g. [9]) that $f$ has $2^{i}$-periodic interval $J_{i}$ (some details are mentioned in the next paragraph). Let

$$
X_{i}=\lim _{\leftarrow}\left\{f^{2^{i}} \mid J_{i}, J_{i}\right\}=\left\{\left(x_{1}, x_{2}, \ldots\right) \mid f^{2^{i}}\left(x_{j+1}\right)=x_{j} \text { and } x_{j} \in J_{i} \text { for all } j>0\right\} .
$$

Then $X_{i}$ is a $2^{i}$-periodic subcontinuum of $I_{f}$ for $\sigma_{f}$.

We are also able to specify shapes of those $2^{i}$-periodic subcontinua. In the next lemma we use the fact (see e.g. [9], p.26 and [7], p.3) that any map $f \in \mathcal{F}$ possesses the unique infinite $\omega$-limit set $\bar{\omega}$ such that

$$
\bar{\omega} \subseteq \bigcap_{i \in \mathbb{N}} \bigcup_{n=1}^{2^{i}} f^{n}\left(J_{i}\right),
$$

where each $J_{i}$ is a nondegenerate $2^{i}$-periodic interval (i.e. $\left.f^{2^{i}}\left(J_{i}\right)=J_{i}\right)$. Intervals $J_{i}$ are called generating. 
Lemma 13 Let $f \in \mathcal{F}$. Then there exists a system $\left\{J_{i}\right\}_{i \geq 0}$ of generating intervals such that, for any $i \geq 0, \lim _{\leftarrow}\left\{\left.f^{2^{i}}\right|_{J_{i}},{ }_{i}\right\}$ is a compactification of a topological ray.

Proof We shall show that there exists a system $\left\{J_{i}\right\}_{i \geq 0}$ of generating intervals such that, for any $i \geq 0$, each $\left.f^{2^{i}}\right|_{J_{i}}$ satisfies assumptions of Theorem 8 . The existence of a system of generating intervals is well known. Within this proof we sketch a construction allowing us to find a particular one that will be useful to us. Let $L_{0}:=\left[u_{0}, v_{0}\right]$ denote the nondegenerate interval for which $f\left(\left[u_{0}, v_{0}\right]\right)=m:=\max \{f(x) \mid x \in I\}$ and $a_{0}$ denote the only fixed point in $\left[v_{0}, 1\right]$. Its existence follows from the ordering of periodic orbits of non-chaotic interval maps [16].

Then for any $i \in \mathbb{N}, f^{-\left(2^{i}\right)}\left(\left[u_{0}, v_{0}\right]\right)$ consists of $2^{2^{i}}$ nondegenerate intervals. Let $L_{i}=\left[u_{i}, v_{i}\right]$ denote the most right one. Then $\left[u_{i}, 1\right] \subseteq f^{2^{i}}\left(\left[u_{i}, 1\right]\right)$ implies the existence of a fixed point $a_{i} \in\left[u_{i}, 1\right]$ of the map $f^{2^{i}}$. It is easy to see that

$$
a_{i-1}<u_{i}<v_{i}<a_{i}<m<1
$$

for any $i \in \mathbb{N}$. Because $f$ is weakly unimodal, the map $f^{2^{i}}$ is weakly unimodal on $\left[a_{i-1}, 1\right]$ as well - namely, $f^{2^{i}}{ }_{\left[a_{i-1}, v_{i}\right]}$ is nondecreasing, $f^{2^{i}}\left(\left[u_{i}, v_{i}\right]\right)=m$ and $\left.f^{2^{i}}\right|_{\left[v_{i}, 1\right]}$ is nonincreasing. Moreover, we can prove $f^{2^{i}}\left(\left[a_{i-1}, m\right]\right)=\left[a_{i-1}, m\right]$. Indeed, because $a_{i-1}$ is a fixed point of $f^{2^{i-1}}$ and $f^{2^{i}}\left(\left[u_{i}, v_{i}\right]\right)=m$ we get $\left[a_{i-1}, m\right] \subseteq f^{2^{i}}\left(\left[a_{i-1}, m\right]\right)$. But if the latter inclusion were strict, the map $\left.f^{2^{i}}\right|_{\left[a_{i}, m\right]}$ would be turbulent (i.e. it would satisfy (2)), which is impossible for zero entropy maps. Thus, $f^{2^{i}}\left(J_{i}\right)=J_{i}$ for $J_{i}:=\left[a_{i-1}, m\right]$.

We claim that the system $\left\{J_{i}\right\}_{i \in \mathbb{N}}$ is a system of generating intervals. By e.g. [9], p.26 and [7], p.3, $m \in \bar{\omega}$ where $\bar{\omega}$ is the unique infinite $\omega$-limit set of $f$. It is well known (e.g. [9]) that $\bar{\omega}$ is contained in the closure of periodic points, thus $\lim _{i \rightarrow \infty} a_{i}=m$. Consequently (3) implies $\bigcap_{i \in \mathbb{N}} J_{i}=\{m\}$.

Because, for any $i \in \mathbb{N}$, the map $\left.f^{2^{i}}\right|_{J_{i}}$ is clearly conjugate to a map $\tilde{f} \in \mathcal{F}$ satisfying $\tilde{f}(0)=$ 0 , Lemma 9 and the proof of Theorem 10 finishes this proof (see Figure 2).

As a consequence of the above we obtain the following result announced in the first section of our paper.

Figure 2 Proof of Lemma 13.

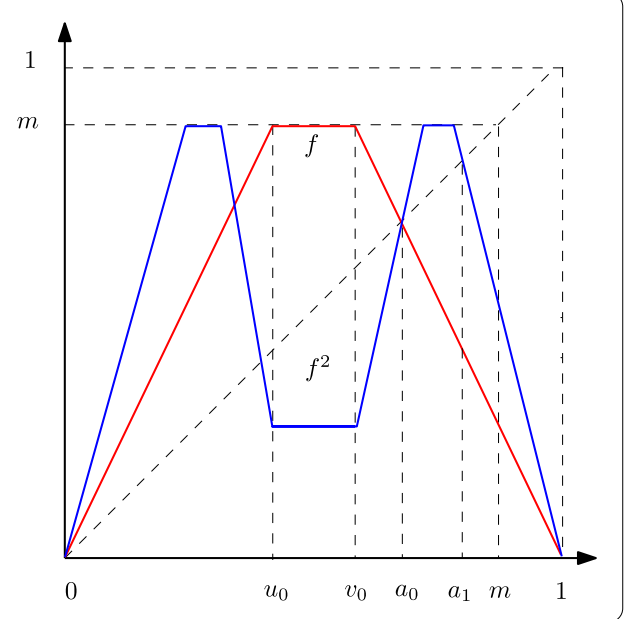


Theorem 3 Suppose $f: I \rightarrow I$ is a Li-Yorke chaotic zero entropy weakly unimodal map. Then $I_{f}$ contains, for every $i$, a subcontinuum $C_{i}$ with the following two properties:

(i) $C_{i}$ is $2^{i}$-periodic under the shift homeomorphism, and

(ii) $C_{i}$ is a compactification of a topological ray.

Proof This follows directly from Lemma 12 and Lemma 13.

Problem 2 Does Lemma 13 hold for any map from $\mathcal{F}$ ?

At the very end of this paper we would like to mention some facts showing that the inverse limit spaces constructed within this work are topologically distinct from those mentioned in [6].

Let $X_{f}=\lim _{\leftarrow}\{f, I\}$ be the (nonchaotic) hereditarily decomposable arc-like attractor from Theorem 8 in [6]; i.e. $f$ is the Delahaye map of type $2^{\infty}$ [23]. By Theorem 5 in [6], the continuum $X_{f}$ is the union of two continua $K_{1}$ and $K_{2}$ such that

(1) $K_{1}$ is homeomorphic to $K_{2}$,

(2) $K_{1}$ is the union of a topological ray $R_{1}$ and $X_{0}^{1}$ that compactifies $R_{1}$; i.e. $\bar{R}_{1} \backslash R_{1}=X_{0}^{1}$,

(3) $K_{2}$ is the union of a topological ray $R_{2}$ and $X_{1}^{1}$ that compactifies $R_{2}$; i.e. $\bar{R}_{2} \backslash R_{2}=X_{1}^{1}$, and

(4) $K_{1} \cap K_{2}=R_{1} \cap R_{2}=\{\hat{p}\}$, where $\hat{p}$ is the fixed point of $\sigma_{f}$.

Consequently, $X_{f}$ is not a compactification of a topological ray (but it is a compactification of a topological line). Therefore no $I_{g}$ given by a weakly unimodal map $g$ in Theorem 1 or Theorem 2 is homeomorphic to $X_{f}$. Now we shall also show that no such $I_{g}$ is homeomorphic to the chaotic hereditarily decomposable arc-like attractor $X_{F}$ from Lemma 9 in [6]; i.e. $X_{F}=\lim _{\leftarrow\{F, I\}}$ and $F$ is the Li-Yorke chaotic modification of Delahaye's map $f$, as described in Section 3 of [6]. Recall that it was shown (Proposition 8 in [6]) that there is a monotone map $\Pi: X_{F} \rightarrow X_{f}$; i.e. $\Pi^{-1}(K)$ is connected for every connected set $K \subseteq X_{f}$. Suppose $X_{F}$ is homeomorphic to $I_{g}$, given by a weakly unimodal map $g$ in Theorem 1 or Theorem 2. Then $X_{F}=\bar{L}$, where $L$ is a topological ray, by Theorem 10. Since $L$ is dense in $X_{f}$ it follows that $\Pi(L)$ is dense and connected in $X_{f}$. It is readily seen that $\Pi(L)$ must contain then the topological line $R=R_{1} \cup R_{2}$. Since $\Pi(L)$ is arcwise connected (as a continuous image of a ray) we must have $\Pi(L)=R$. It follows that $\Pi \mid L: L \rightarrow R$ is a monotone map of $[0,+\infty)$ onto $(-\infty,+\infty)$ leading to a contradiction.

We also note that each our attractor has an absolute end point; i.e. a point at which it is locally connected and which does not separate it (see [24] for more on absolute end points). However, the attractor $X_{f}$ from [6] does not have such a point.

The above remarks lead to the following questions.

Problem 3 Suppose $g$ is a Li-Yorke chaotic weakly unimodal map of type $2^{\infty}$. Is $I_{g}$ homeomorphic to a ray limiting onto one of the attractors described in [6] or a subcontinuum of one of them?

Problem 4 Suppose $f$ and $g$ are two Li-Yorke chaotic weakly unimodal maps of type $2^{\infty}$ that are in two different differentiability classes, as guaranteed by Theorem 1 and Theorem 2. Are $I_{f}$ and $I_{g}$ homeomorphic? 
One should note that for an arbitrary nondegenerate metric continuum $P$, there is an uncountable family of topologically distinct metric compactifications of a topological ray $L$, such that $\bar{L} \backslash L=P[25]$.

\section{Competing interests}

The authors declare that they have no competing interests.

\section{Authors' contributions}

Both authors contributed equally to the writing of this paper. Both authors read and approved the final manuscript.

\section{Acknowledgements}

The authors express many thanks to Henk Bruin and Sonja Štimac for the helpful feedback during the first author's visit in Faculty of Mathematics at University of Vienna in May 2014. This author is grateful for the kind hospitality of the faculty during his stay in Vienna and acknowledges financial support of the DFG network grant Oe 538/3-1, OeAD and the Polish Ministry of Science and Higher Education. This work was supported by the European Regional Development Fund in the IT4Innovations Centre of Excellence project (CZ.1.05/1.1.00/02.0070). J Boroński also gratefully acknowledges the partial support from the MSK DT1 Support of Science and Research in the Moravian-Silesian Region 2013 (RRC/05/2013).

\section{Endnote}

a The authors are grateful to Henk Bruin for bringing this fact to their attention.

\section{Received: 12 March 2015 Accepted: 6 July 2015 Published online: 26 July 2015}

\section{References}

1. Bell, H: A fixed point theorem for plane homeomorphism. Fundam. Math. 100, 119-128 (1978)

2. Cartwright, ML, Littlewood, JE: Some fixed point theorems. With appendix by HD Ursell. Ann. Math. (2) 54, 1-37 (1951)

3. Barge, M, Martin, J: Chaos, periodicity, and snakelike continua. Trans. Am. Math. Soc. 289(1), 355-365 (1985)

4. Barge, M, Martin, J: The construction of global attractors. Proc. Am. Math. Soc. 110(2), $523-525$ (1990)

5. Barge, M, Diamond, B: The dynamics of continuous maps of finite graphs through inverse limits. Trans. Am. Math. Soc. 344(2), 773-790 (1994)

6. Boroński, JP, Oprocha, P: On indecomposability in chaotic attractors. Proc. Am. Math. Soc. 143(8), 3659-3670 (2015)

7. Misiurewicz, M, Smítal, J: Smooth chaotic maps with zero topological entropy. Ergod. Theory Dyn. Syst. 8, 421-424 (1988)

8. Ingram, WT: Periodicity and indecomposability. Proc. Am. Math. Soc. 123, 1907-1916 (1995)

9. Blokh, A: The spectral decomposition for one-dimensional maps. arXiv:math/9201290v1

10. Engelking, R: Zarys Topologii Ogólnej (Polish) [Outline of General Topology]. Biblioteka Matematyczna, vol. 25. Państwowe Wydawnictwo Naukowe, Warsaw (1965)

11. Lewis, W: The pseudo-arc. In: Continuum Theory and Dynamical Systems. Contemporary Mathematics, vol. 117, pp. 103-123 (1991)

12. Chen, L, Li, SH: Dynamical connections between a continuous map and its inverse limit space. In: Continuum Theory and Dynamical Systems. Lecture Notes in Pure and Appl. Math., vol. 149, pp. 89-97. Dekker, New York (1993)

13. Bowen, R: Entropy for group endomorphism and homogeneous spaces. Trans. Am. Math. Soc. 153, 401-414 (1971)

14. Du, B: Smooth weakly chaotic interval maps with zero topological entropy. In: Dynamical Systems and Related Topics (Nagoya, 1990). Adv. Ser. Dynam. Systems, vol. 9, pp. 72-79. World Scientific, River Edge (1991)

15. López, VJ: An explicit description of all scrambled sets of weakly unimodal functions of type $2^{\infty}$. Real Anal. Exch. 21 , 664-688 (1995/96)

16. Block, LS, Coppel, WA: Dynamics in One Dimension. Lecture Notes in Mathematics. Springer, Berlin (1992)

17. Misiurewicz, M: Structure of mappings of an interval with zero entropy. Publ. Math. Inst. Hautes Études Sci. 53, 5-16 (1981)

18. Jiménez López, V, Snoha, Ł: All maps of type $2^{\infty}$ are boundary maps. Proc. Am. Math. Soc. 125(6), 1667-1673 (1997)

19. Bruin, H, Štimac, S: Entropy of homeomorphisms on unimodal inverse limit spaces. Nonlinearity 26, 991-1000 (2013)

20. Lü, J, Xiong, J, Ye, X: The inverse limit space and the dynamics of a graph map. Topol. Appl. 107(3), 275-295 (2000)

21. Minc, P, Transue, WRR: Accessible points of hereditarily decomposable chainable continua. Trans. Am. Math. Soc. 332(2), 711-727 (1992)

22. Henderson, GW: The pseudo-arc as an inverse limit with one binding map. Duke Math. J. 31, 421-425 (1964)

23. Delahaye, J-P: Fonctions admettant des cycles d'ordre n'importe quelle puissance de 2 et aucun autre cycle (French) [Functions admitting cycles of any power of 2 and no other cycle]. C. R. Acad. Sci. Paris, Sér. A-B 291(4), A323-A325 (1980)

24. Rosenholtz, I: Absolute endpoints of chainable continua. Proc. Am. Math. Soc. 103(4), 1305-1314 (1988)

25. Martínez-de-la-Vega, V, Minc, P: Uncountable families of metric compactifications of the ray. Topol. Appl. 173(15), 28-31 (2014) 